\title{
Hall noise and transverse freezing in driven vortex lattices
}

\author{
Alejandro B. Kolton and Daniel Domínguez \\ Centro Atómico Bariloche, 8400 S. C. de Bariloche, Rio Negro, Argentina \\ Niels Grønbech-Jensen \\ Theoretical Division, Los Alamos National Laboratory, Los Alamos, NM 87545, USA
}

(May 22, 2021)

\begin{abstract}
We study driven vortices lattices in superconducting thin films. Above the critical force $F_{c}$ we find two dynamical phase transitions at $F_{p}$ and $F_{t}$, which could be observed in simultaneous noise measurements of the longitudinal and the Hall voltage. At $F_{p}$ there is a transition from plastic flow to smectic flow where the voltage noise is isotropic (Hall noise $=$ longitudinal noise) and there is a peak in the differential resistance. At $F_{t}$ there is a sharp transition to a frozen transverse solid where the Hall noise falls down abruptly and vortex motion is localized in the transverse direction.
\end{abstract}

PACS numbers: 74.60.Ge, 74.40.+k, 05.70.Fh

The study of the collective motion of vortex lattices in superconductors has brought new concepts in the nonequilibrium statistical physics of driven disordered media [1] 18]. The prediction [1] of a dynamical phase transition upon increasing drive, from a fluidlike plastic flow regime [2 4] to a coherently moving solid [1], has motivated an outburst of recent theoretical [5 [7, experimental 811, and simulation 12 18 work. The relevant physics of the high velocity driven phase is controlled by the transverse displacements (in the direction perpendic-

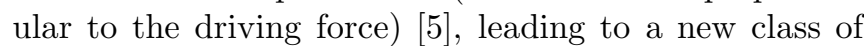
driven systems characterized by anisotropic spatial structures with transverse periodicity [5] 7]. Recently, these moving anisotropic vortex structures have been observed experimentally by Pardo et al. [11], and their different features have been studied in 2D [12 16] and 3D [17, 18 simulations. In this letter, we show that a better insight on the moving phases can be obtained from studying the anisotropic temporal fluctuations. We find two dynamical phase transitions which could be observed experimentally by measuring voltage noise [19,20] both in the longitudinal and transversal directions. In the transverse direction, where the system is not driven, we can study diffusion, from which we define an effective temperature in analogy with equilibrium physics [1, ]7].

The equation of motion of a vortex in position $\mathbf{r}_{i}$ is:

$$
\eta \frac{d \mathbf{r}_{i}}{d t}=-\sum_{j \neq i} \nabla_{i} U_{v}\left(r_{i j}\right)-\sum_{p} \nabla_{i} U_{p}\left(r_{i p}\right)+\mathbf{F},
$$

where $r_{i j}=\left|\mathbf{r}_{i}-\mathbf{r}_{j}\right|$ is the distance between vortices $i, j, r_{i p}=\left|\mathbf{r}_{i}-\mathbf{r}_{p}\right|$ is the distance between the vortex $i$ and a pinning site at $\mathbf{r}_{p}, \eta=\frac{\Phi_{0} H_{c 2} d}{c^{2} \rho_{n}}$ is the Bardeen-Stephen friction and $\mathbf{F}=\frac{d \Phi_{0}}{c} \mathbf{J} \times \mathbf{z}$ is the driving force due to an applied current $\mathbf{J}$. A two-dimensional superconductor is realized in thin films of thickness $d$ where $d \ll \lambda$, which have an effective penetration depth $\Lambda=2 \lambda^{2} / d$. Since $\Lambda$ is of the order of the sample size $(\Lambda \approx 200 \mu m$ in $[9])$, the vortex-vortex interaction is logarithmic: $U_{v}(r)=-A_{v} \ln (r / \Lambda)$, with $A_{v}=\Phi_{0}^{2} / 8 \pi \Lambda$ [3, 13].
The vortices interact with a random uniform distribution of attractive pinning centers with $U_{p}(r)=-A_{p} e^{-(r / \xi)^{2}}$ with $\xi$ being the coherence length. We normalize length scales by $\xi$, energy scales by $A_{v}$, and time is normalized by $\tau=\eta \xi^{2} / A_{v}$. We consider $N_{v}$ vortices and $N_{p}$ pinning centers in a rectangular box of size $L_{x} \times L_{y}$, and the normalized vortex density is $n_{v}=N_{v} \xi^{2} / L_{x} L_{y}=B \xi^{2} / \Phi_{0}$. Moving vortices induce a total electric field $\mathbf{E}=\frac{B}{c} \mathbf{v} \times \mathbf{z}$, with $\mathbf{v}=\frac{1}{N_{v}} \sum_{i} \mathbf{v}_{i}$.

We study the dynamical regimes in the velocity-force (voltage-current) characteristics at $T=0$, solving Eq.(1) for increasing values of $\mathbf{F}=F \mathbf{y}$. We consider a constant vortex density $n_{v}=0.12$ in a box with $L_{x} / L_{y}=\sqrt{3} / 2$, and $N_{v}=64,144,196,256,400,784$ (we show results for $\left.N_{v}=400\right)$. We take a pinning strengh of $A_{p} / A_{v}=0.2$ with a density of pinning centers $n_{p}=0.24$. We use periodic boundary conditions and the periodic long-range logarithmic interaction is evaluated with an exact and fast converging sum [21]. The equations are integrated with a time step of $\Delta t=0.01 \tau$ and averages are evaluated in 32768 integration steps after 2000 iterations for equilibration (when the total energy reaches a stationary value). Each simulation is started at $F=0$ with an ordered triangular lattice and slowly increasing the force in steps of $\Delta F=0.05$ up to values as high as $F=8$.

First we start by looking at the vortex trajectories and their translational order in the steady state phases as was done in 12 18]. In Figure 1(a-c) we show the vortex trajectories $\left\{\mathbf{r}_{i}(t)\right\}$ for typical values of $F$ by plotting all the positions of the vortices for all the time iteration steps. We also study the time-averaged structure factor $S(\mathbf{k})=\left\langle\left|\frac{1}{N_{v}} \sum_{i} \exp \left[i \mathbf{k} \cdot \mathbf{r}_{i}(t)\right]\right|^{2}\right\rangle$, which is shown in Fig. 1(d-f). In Fig. 2(a) we plot the average vortex velocity $V=\left\langle V_{y}(t)\right\rangle=\left\langle\frac{1}{N_{v}} \sum_{i} \frac{d y_{i}}{d t}\right\rangle$, in the direction of the force as a function of $F$ and its corresponding derivative $d V / d F . \quad\left(V=E / \rho_{f} J_{0}\right.$ with $\rho_{f}$ the flux flow resistivity and $J_{0}=c A_{v} / d \xi \Phi_{0}$, therefore $d V / d F$ is proportional to the differential resistivity $\left.d V / d F=\rho_{f}^{-1} d E / d J\right)$. Below a critical force $F_{c} \approx 0.25$ all the vortices are pinned and 

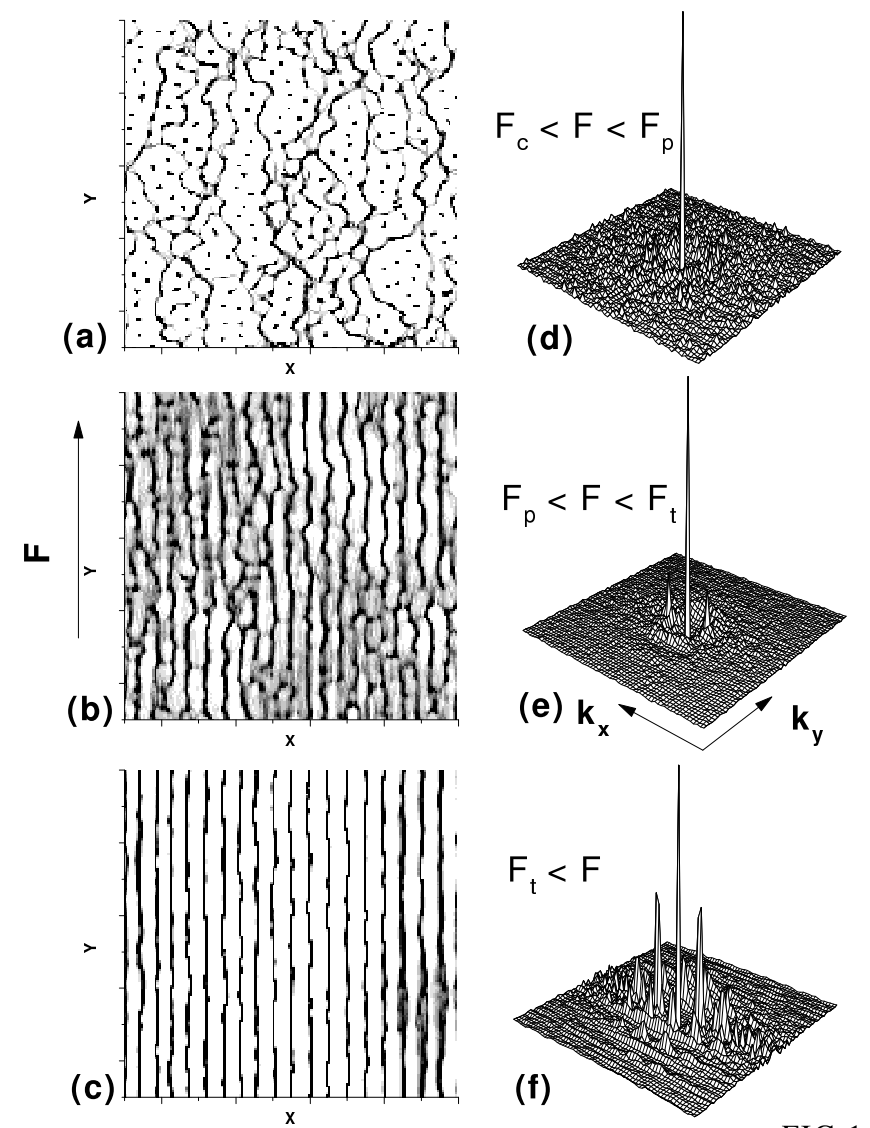

FIG. 1. Vortex trajectories: (a) $F=0.5$, (b) $F=2.5$, (c) $F=7$. Surface intensity plot of the averged structure factor $S(\mathbf{k}):(\mathrm{d}) F=0.5,(\mathrm{e}) F=2.5$, (f) $F=7$.

there is no motion, $V=0$. Above $F_{c}$, we distinguish between three different dynamical regimes:

(i) Plastic flow: $F_{c}<F<F_{p}$. At $F_{c}$ vortices start to move in a few filamentary channels, as was also seen in [3]. A typical situation is shown in Fig. 1(a), where a fraction of the vortices are moving in an intrincate network of channels. As the force is increased a higher fraction of vortices is moving. In this regime, vortices can move in the transverse direction (perpendicular to $\mathbf{F}$ ) through the tortuous structure of channels [4]. We see in Fig. 1(d) that $S(\mathbf{k})$ only has the central peak showing the absence of ordering in this plastic flow regime 12 .

(ii) Smectic flow: $F_{p}<F<F_{t}$. We observe a peak in the differential resistance at a characteristic force $F_{p} \approx 1.3$. At $F=F_{p}$ we see that all the vortices are moving in a seemingly isotropic channel network with maximum interconnectivity. In other simulations the peak in the differential resistance was found to coincide with a maximum in the number of defects 115 and with the onset of orientational order [13]. Also, the value of $F_{p}$ was taken in the experiment of Hellerqvist et al [9] as an indication of a dynamical phase transition. In fact, we find that above $F_{p}$ a new dynamic regime sets in. In this case, as we show in Fig. 1(b), all the vortices are moving

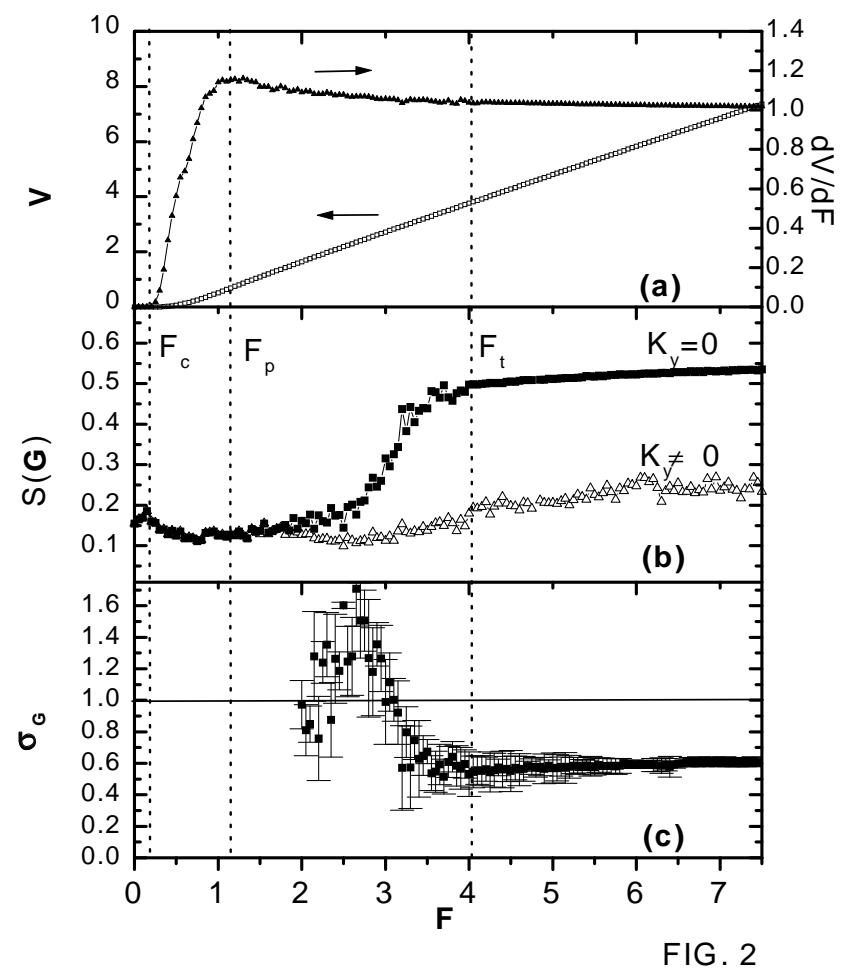

FIG. 2. (a) Velocity-force curve (voltage-current characteristics), left scale, white points. $d V / d F$ curve (differential resistance), right scale, black points. (b) Intensity of the Bragg peaks. For smectic ordering, $S\left(G_{1}\right), K_{y}=0$ : black points. For longitudinal ordering, $S\left(G_{2,3}\right), K_{y} \neq 0$ : white points. (c) Finite size exponent $\sigma_{G}\left(S\left(G_{1}\right) \sim N_{v}^{-\sigma_{G}}\right)$.

in trajectories that are mostly parallel to the force, forming "elastic channels". Two small Bragg peaks appear in the structure factor along the $K_{y}=0$ axis, as seen in Fig. 1(e), which correspond to $\mathbf{G}_{1}=\left( \pm 2 \pi / a_{0}, 0\right)$. This is consistent with the onset of "smectic" ordering [6, 12 in the transverse direction with elastic channels separated by a distance $\sim a_{0}=n_{v}^{-1 / 2}$. In this regime the transverse motion consists in vortex jumps from one channel to another, resembling "thermally" activated transitions induced by local chaos. The rate of these transitions decreases with increasing force, and they correspond to the permeation modes discussed in [6]. In Fig. 2(b) we plot the magnitude of the Bragg peaks at $G_{1}, S_{s}=S\left(G_{1}\right)$, corresponding to smectic ordering $\left(K_{y}=0\right)$, and the other neighbouring peaks at $\mathbf{G}_{2}=$ $\pm 2 \pi / a_{0}(1 / 2, \sqrt{3} / 2)$ and $\mathbf{G}_{3}= \pm 2 \pi / a_{0}(-1 / 2, \sqrt{3} / 2)$, $\left.S_{l}=\left(S\left(G_{2}\right)+S\left(G_{3}\right)\right) / 2\right)$, corresponding to longitudinal ordering $\left(K_{y} \neq 0\right)$. We see that above $F_{p}$ the intensity of the smectic peak $S_{s}$ starts to grow and $S_{s} \gg S_{l}$, while below $F_{p}$ the spatial structure is isotropic, $S_{s}=S_{l} \ll 1$. The Bragg peak heights depend with system size as $S(G) \sim N_{v}^{-\sigma_{G}}$, where $\sigma_{G}=0$ means long-range or- 
der (LRO), $0<\sigma_{G}<1$ means quasi long-range order (QLRO) and $\sigma_{G}=1$ means short-range order (SRO). In Fig. 2(c) we show the corresponding results for the smectic peak $S_{s}=S\left(G_{1}\right)$ for sizes $N_{v}=256,400,784$. We see that $\sigma_{G} \gtrsim 1$ in this regime: there is only short range smectic order, thus this phase corresponds to a liquid. In this sense the transition at $F_{p}$ is a dynamic transition in the flow, as found in [16] for strong disorder $\left(n_{p}=1\right)$. When approaching $F_{t}$ (see below) we see a precursor of QLRO $\left(\sigma_{g}<1\right)$ but with strong fluctuations.

(iii) Frozen transverse solid: $F>F_{t}$. At a new characteristic force $F_{t}$, the jumps between channels suddenly stop and vortex motion becomes frozen in the direction perpendicular to $\mathbf{F}$. An example for $F>F_{t}$ is shown in Fig. 1(c) where we see well defined elastic channels parallel to $\mathbf{F}$. The corresponding structure factor is in Fig. 1(e) where new peaks appear in $S(\mathbf{k})$ in directions with $K_{y} \neq 0$, like $G_{2}, G_{3}$, showing that there is some longitudinal ordering between the channels. These extra peaks are smaller than the smectic peaks, and $S(\mathbf{k})$ is very anisotropic. We note in Fig. 2(a) that $F_{t}$ is the point where the noisy behavior in $d V / d F$ ceases. A simialar criterion was used by Bhattacharya and Higgins to define their dynamical phase diagram [8]. In Fig. 2(b) we see that in $F_{t}$ there is an increase in the longitudinal ordering $S_{l}$, and both $S_{s}$ and $S_{l}$ tend to saturate at an almost constant value for $F \gg F_{t}$. In Fig. 2(c) we find that there is smectic QLRO [5.,6, 12 with a value of $0.5<\sigma_{G}<0.7$. However, we were not able to obtain a reliable finite size analysis for the longitudinal peaks since they have large fluctuations from size to size. Also, we find hysteresis in $S_{s}$ around $F_{t}$ when decreasing $F$.

A better understanding of the dynamical transitions can be obtained from studying the temporal behavior of the system in both directions. It has been observed experimentally that the longitudinal voltage can show low frequency noise [19,20]. This voltage noise reaches a very large value above the critical current, which has been attributed to plastic flow [20], and then the noise decreases for large current. In addition, even when the total dc transverse voltage $\left\langle V_{x}\right\rangle=\left\langle\frac{1}{N_{v}} \sum_{i} \frac{d x_{i}}{d t}\right\rangle$ is zero, it can also have fluctuations and noise [22]. In fact, it is easy to understand that this Hall noise will be closely related to the wandering and wiggling of the plastic flow channels and to the jumps between elastic channels in the smectic phase. We have calculated the power spectrum of both the longitudinal voltage, $S_{y}(f)=\left|\frac{1}{T} \int_{0}^{T} d t\left(V_{y}(t)-V\right) \exp (i 2 \pi f t)\right|^{2}$, and the transverse voltage, $S_{x}(f)=\left|\frac{1}{T} \int_{0}^{T} d t V_{x}(t) \exp (i 2 \pi f t)\right|^{2}$. The low frequency noise is defined as $P_{x, y}=\lim _{f \rightarrow 0} S_{x, y}(f)$. In Fig. 3(a) we show the values of the longitudinal noise $P_{y}$ and the Hall noise $P_{x}$ as a function of the force $\left(P_{x, y}\right.$ were approximated from the average of the 10 lowest frequencies). We see that near the critical force, the longitudinal noise is large while the Hall noise is one order

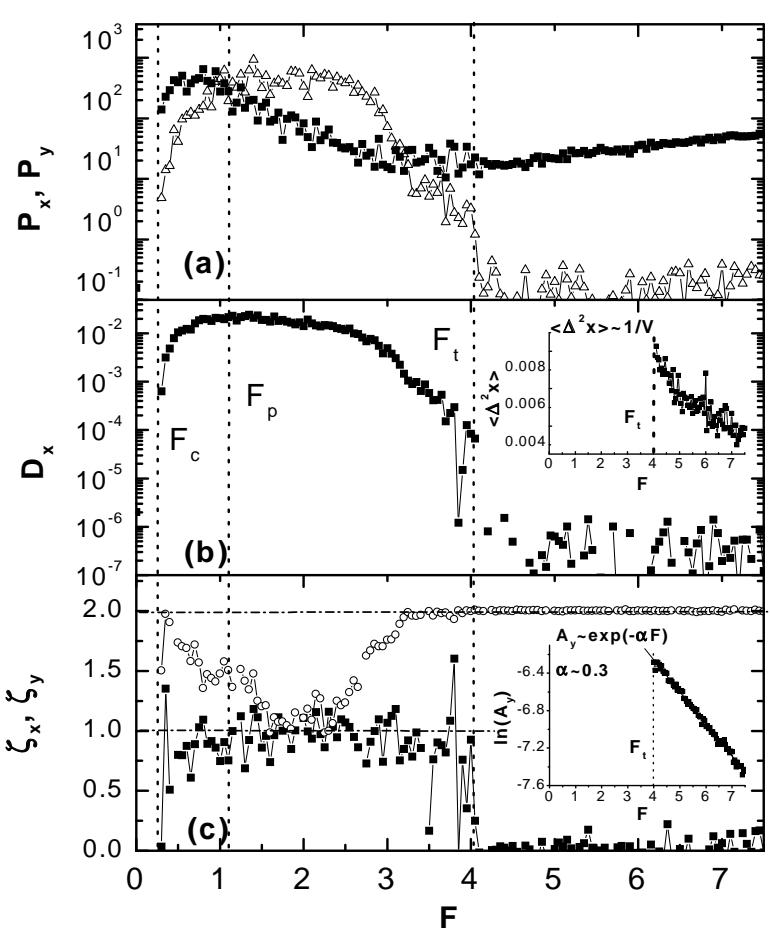

FIG. 3

FIG. 3. (a) Low frequency voltage noise vs $F$. Longitudinal voltage noise, $P_{y}$, black points. Hall noise, $P_{x}$, white points. (b) Diffusion coefficient for transversal motion, $D_{x}$. The inset shows the average transverse quadratic displacement $\left\langle\Delta^{2} x\right\rangle$ in the frozen phase, $F>F_{t}$. (c) Diffusion exponents $\zeta_{x}$ (black dots) and $\zeta_{y}$ (white dots) defined from: $\left\langle[\tilde{x}(t)-\tilde{x}(0)]^{2}\right\rangle \sim t^{\zeta_{x}}$, $\left\langle[\tilde{y}(t)-\tilde{y}(0)]^{2}\right\rangle \sim t^{\zeta_{y}}$. The inset shows a plot of $A_{y}$ defined from a fit $\left\langle[\tilde{y}(t)-\tilde{y}(0)]^{2}\right\rangle=A_{y} t^{2}$ for $F>F_{t}$.

of magnitude smaller. In this regime of plastic flow the noise is dominated by fluctuations in the direction of motion and by channel tortuosity [4], and since there are few channels the Hall noise is small. When the number of channels increases the vortices wander more in the $x$ direction and the Hall noise increases. At $F_{p}$ the voltage noise becomes isotropic, $P_{x}=P_{y}$. This is the point where we have seen the highest interconnection in the channel network. Above $F_{p}$, the onset of elastic channels and smectic ordering reduces the longitudinal noise. On the other hand, the Hall noise remains large due to the "activated" jumps between elastic channels. At $F_{t}$ the Hall noise falls abruptly, nearly two orders of magnitude. This corresponds to a freezing transition of vortex motion in the transverse direction. Above $F_{t}$ there are no more vortex jumps between elastic channels. The low frequency noise can be closely related to diffusive motion for large times. We analyze the average quadratic displacements of vortices in both directions from their center of mass position $\left(X_{c m}(t), Y_{c m}(t)\right)$ as a function of time. We define $w_{x}(t)=\frac{1}{N_{v}} \sum_{i}\left[\tilde{x}_{i}(t)-\tilde{x}_{i}(0)\right]^{2}$, and $w_{y}(t)=$ 
$\frac{1}{N_{v}} \sum_{i}\left[\tilde{y}_{i}(t)-\tilde{y}_{i}(0)\right]^{2}$ where $\tilde{x}_{i}(t)=x_{i}(t)-X_{c m}(t)$, $\tilde{y}_{i}(t)=y_{i}(t)-Y_{c m}(t)$. We observe that the vortex motion for $F_{c}<F<F_{t}$ is diffusive in the transverse direction, $w_{x}(t) \sim D_{x} t$. In Fig. 3(b) we show the behavior of the transverse diffusion coefficient $D_{x}$. It closely follows the behavior of the Hall noise, $D_{x} \propto P_{x}$. The transverse diffusion is maximum at the peak in the differential resistance, $F_{p}$, and it has an abrupt jump to zero at $F_{t}$ indicating the transverse freezing transition. It is interesting to note that melting transtions also show a jump in the diffusion coefficient. Above $F_{t}$, the transverse wandering is independent of time since vortex motion is localized in the $x$ direction: $w_{x}(t) \approx\left\langle\Delta^{2} x\right\rangle$. In the inset of Fig. 3(b) we show $\left\langle\Delta^{2} x\right\rangle$ vs. $F$ for $F>F_{t}$. We find that $\left\langle\Delta^{2} x\right\rangle \approx 0.02 a^{2}$ at $F_{t}$, consistent with a Lindemann criterion for melting [7]. Since in equibrium the diffusion coefficient $D$ is proportional to $T$, we interpret $D_{x}$ as an indication of an "effective temperature" $T_{\text {eff }}$. This means that $T_{\text {eff }}$ raises above the critical force, has a maximum at the peak in the differential resistance and decreases when the motion starts to order. Following the same idea, above $F_{t}$ we can interpret $\left\langle\Delta^{2} x\right\rangle$ as proportional to $T_{\text {eff }}$. Here we find that $\left\langle\Delta^{2} x\right\rangle$ decreases with $F$, consistent with a $T_{\text {eff }} \sim 1 / V$ behavior [1]7].

The long-time behavior is better understood by studying the diffusion exponents $w_{x}(t) \sim t^{\zeta_{x}}$ and $w_{y}(t) \sim t^{\zeta_{y}}$. In Fig. 3(c) we show the behavior of $\zeta_{x, y}$. We see that for $F_{c}<F<F_{t}, \zeta_{x} \approx 1$ corresponding to normal diffusion, while for $F>F_{t}, \zeta_{x} \approx 0$ corresponding to the freezing of transverse motion. More interestingly, the motion in the longitudinal direction is always superdiffusive, $\zeta_{y}>1$. Above $F_{t}$, in the frozen phase, the longitudinal fluctuations become ballistic, $\zeta_{y} \approx 2$. Since the vortex positions are localized in the $x$ direction, we see that $w_{y}(t)=\left\langle(y(t)-y(0))^{2}\right\rangle \approx A_{y} t^{2}$, with $A_{y}=\left\langle\Delta^{2} v_{y}\right\rangle$ the dispersion of the average velocities of the elastic channels. In the inset of Fig. 3(c) we find that $A_{y}$ decreases exponentially with the force $A_{y} \sim \exp (-\alpha F)$. [This suggests a "thermal" distribution of channel velocities, since $T_{\text {eff }} \sim 1 / F$, then $\left.\left\langle\Delta^{2} v_{y}\right\rangle \sim \exp \left(-\alpha^{\prime} / T_{\text {eff }}\right)\right]$. In the smectic flow region an exponent $1<\zeta_{y}<2$ can be explained from assuming that vortices move most of the time in the elastic channels and occasionally have diffusive jumps between channels in the transverse direction.

In conclusion, we have obtained evidence of two dynamical phase transitions which can be verified experimentally by measurements of voltage noise and Hall noise. The first transition at $F_{p}$ is the point of isotropic noise and maximum transverse diffusion (i.e., maximum effective temperature) and corresponds to the observed peak in the differential resistance. The second transition at $F_{t}$ is a freezing transition in the transverse direction, where the transverse diffusion vanishes abruptly and the Hall noise drops many orders of magnitude.

We acknowledege discussions with A. R. Bishop, P. Cornaglia, Y. Fasano, F. de la Cruz, S. Bhattacharya,
V. B. Geshkenbein, F. Pardo, V. M. Vinokur, M. B. Weissman, and G. Zimányi. This work has been supported by a grant from ANPCYT (Argentina). D. D. and A.B.K. also acknowledge support from Fundación Antorchas, Conicet and CNEA (Argentina). Parts of this work were performed under the auspices of the U.S. D.o.E.

[1] A. E. Koshelev and V. M. Vinokur, Phys. Rev. Lett. 73, 3580 (1994).

[2] H. J. Jensen et al., Phys. Rev. Lett. 60, 1676 (1988); A.-C. Shi and A. J. Berlinsky, Phys. Rev. Lett. 67, 1926 (1991); A. E. Koshelev, Physica C 198, 371 (1992); M. C. Faleski et al., Phys. Rev. B 54, 12427 (1996); C. Reichhardt et al., Phys. Rev. Lett. 78, 2648 (1997).

[3] N. Grønbech-Jensen, A. R. Bishop and D. Domínguez, Phys. Rev. Lett. 76, 2985 (1996).

[4] C. J. Olson, C. Reichhardt and F. Nori, Phys. Rev. Lett. 80, 2197 (1998).

[5] T. Giamarchi and P. Le Doussal, Phys. Rev. Lett. 76, 3408 (1996); P. Le Doussal and T. Giamarchi, Phys. Rev. B 57, 11356 (1998).

[6] L. Balents, M. C. Marchetti and L. Radzihovsky, Phys. Rev. B 57, 7705 (1998).

[7] S. Scheidl and V. M. Vinokur, Phys. Rev. E 57, 2574 (1998); Phys. Rev. B 57, 13800 (1998); Phys. Rev. B 56, R8522 (1997); I S. Aranson, S. Scheidl and V. M. Vinokur, Phys. Rev. B 58, 14541 (1998).

[8] S. Bhattacharya and M. J. Higgins, Phys. Rev. Lett. 70, 2617 (1993); Phys. Rev. B 52, 64 (1995). M. J. Higgins and S. Bhattacharya, Physica C 257, 232 (1996).

[9] M. C. Hellerqvist et al., Phys. Rev. Lett. 76, 4022 (1996).

[10] U. Yaron et al., Nature (London) 376, 743 (1995).

[11] F. Pardo et al, Nature (London) 396, 348 (1998).

[12] K. Moon, R. T. Scalettar and G. Zimányi, Phys. Rev. Lett. 77, 2778 (1996).

[13] S. Ryu et al., Phys. Rev. Lett. 77, 5114 (1996).

[14] S. Spencer and H. J. Jensen, Phys. Rev. B 55, 8473 (1997).

[15] C. J. Olson, C. Reichhardt and F. Nori, Phys. Rev. Lett. 81, 3757 (1998).

[16] D. Domínguez, Phys. Rev. Lett. 82, 181 (1999).

[17] D. Domínguez, N. Grønbech-Jensen and A.R. Bishop, Phys. Rev. Lett. 78, 2644 (1997).

[18] I. Aranson, A. Koshelev and V. Vinokur, Phys. Rev. B 56, 5136 (1997).

[19] W. J. Yeh and Y. H. Kao, Phys. Rev. B 44, 360 (1991).

[20] A. C. Marley et al., Phys. Rev. Lett. 74, 3029 (1995); R. D. Merithew et al., Phys. Rev. Lett. 77, 3197 (1996); M. W. Rabin et al, Phys. Rev. B 57, R720 (1998); T. Tsuboi et al, Phys. Rev. Lett. 80, 4550 (1998).

[21] N. Grønbech-Jensen, Int. J. Mod. Phys. C 7, 873 (1996); Comp. Phys. Comm. 118, (1999), in press.

[22] N. Grønbech-Jensen et al., Phys. Rev. B 46, 11149 (1992). 